\title{
Avaliação da disposição para o perdão em pacientes com hipertensão arterial sistêmica
}

\author{
Evaluation of the forgiveness disposition in patients with systemic arterial hypertension \\ Evaluación de la disposición del perdón en pacientes con hipertensión arterial sistémica
}

Recebido: 06/08/2021 | Revisado: 15/08/2021 | Aceito: 17/08/2021 | Publicado: 20/08/2021

Milena Rabelo de Souza
ORCID: https://orcid.org/0000-0003-3005-8070
Universidade Federal de Sergipe, Brasil
E-mail: milenarabelo32@ gmail.com
Thais Costa Mendonça
ORCID: https://orcid.org/0000-0002-1842-443X
Universidade Federal de Sergipe, Brasil
E-mail: thaiscostaa13@ @otmail.com
Ana Carla Ferreira Silva dos Santos
ORCID: https://orcid.org/0000-0003-3616-8967
Universidade Federal de Sergipe, Brasil
E-mail: carlafss@ yahoo.com.br
Viviane Ferreira Silva dos Santos
ORCID: https://orcid.org/0000-0003-2303-8768
Universidade Federal de Sergipe, Brasil
E-mail: vivianefss @ gmail.com
Carla Kalline Alves Cartaxo Freitas
ORCID: https://orcid.org/0000-0001-7604-9132
Universidade Federal de Sergipe, Brasil
E-mail: carlakalline@ gmail.com
Karenine Maria Holanda Cavalcante
ORCID: https://orcid.org/0000-0003-0840-6833
Universidade Federal de Sergipe, Brasil
E-mail: karenineholanda@ gmail.com
Diego dos Passos Santiago
ORCID: https://orcid.org/0000-0002-0000-3362
Universidade Federal de Sergipe, Brasil
E-mail: diegosanttiago@ @otmail.com

\section{Resumo}

Objetivo: avaliar a disposição para perdoar em indivíduos com hipertensão arterial sistêmica. Metodologia: trata-se de um estudo transversal e quantitativo com abordagem descritiva e analítica. O período de coleta ocorreu entre março de 2020 e janeiro de 2021. Participaram da pesquisa 81 hipertensos assistidos pela clínica de saúde da família "Dr. Davi Marcos de Lima". Os envolvidos foram convidados a responder um questionário contendo dados sociodemográficos e a Escala de Disposição para Perdoar. Além disso, para análise estatística considerou-se o nível de significância de 95\%. Resultados: a amostra compôs-se de 81 indivíduos. No presente estudo, houve prevalência do sexo feminino (66,7\%), idade superior ou igual a 60 anos (63\%) e 53,7\% possuíam Ensino Fundamental incompleto. Houve predominância de indivíduos casados 50,6\%; 66,7\% vivem com o companheiro, 29,6\% residem apenas com uma pessoa. A maioria dos indivíduos $69,1 \%$ não trabalham e/ou estão aposentados. A religião predominante foi a católica com 74,1\%. Correlacionando os dados sociodemográficos com a escala do perdão as variáveis de estado civil e quantidade de pessoas que residiam com o hipertenso foram significantes ( $\mathrm{p}$-valor <0,005). Conclusão: Percebe-se, com esse estudo, que os hipertensos se demostraram dispostos a perdoar. Além disso, o estudo mostrou que estado civil "casado" e "outros" interferem na disposição que o indivíduo tem de perdoar e que os hipertensos que residem com um a três indivíduos estão dispostos a perdoar mais.

Palavras-chave: Perdão; Hipertensão; Atenção primária à saúde; Enfermeiras e enfermeiros.

\section{Abstract}

Objective: To assess the willingness to forgive in individuals with systemic arterial hypertension. Methodology: this is a cross-sectional and quantitative study with a descriptive and analytical approach. The collection period took place between March 2020 and January 2021. 81 hypertensive patients assisted by the family health clinic "Dr. Davi Marcos de Lima". Those involved were invited to answer a questionnaire containing sociodemographic data and the Willingness to Forgive Scale. In addition, for statistical analysis, a significance level of $95 \%$ was considered. Results: the sample consisted of 81 individuals. In the present study, there was a prevalence of females (66.7\%), aged over or equal to 60 years $(63 \%)$ and $53.7 \%$ had incomplete Elementary School. There was a predominance of married 
individuals $50.6 \%$; $66.7 \%$ live with a partner, $29.6 \%$ live with only one person. The majority of individuals $69.1 \%$ do not work and/or are retired. The predominant religion was Catholic with $74.1 \%$. Correlating the sociodemographic data with the forgiveness scale, the variables of marital status and number of people living with the hypertensive were significant ( $\mathrm{p}$-value $<0.005$ ). Conclusion: With this study, it can be seen that hypertensive patients were willing to forgive. In addition, the study showed that marital status "married" and "others" interfere with the individual's willingness to forgive and that hypertensive individuals who live with one to three individuals are willing to forgive more.

Keywords: Forgiveness; Hypertension; Primary health care; Nurses and nurses.

\section{Resumen}

Objetivo: Evaluar la disposición a perdonar en personas con hipertensión arterial sistémica. Metodología: se trata de un estudio transversal y cuantitativo con enfoque descriptivo y analítico. El período de recogida se llevó a cabo entre marzo de 2020 y enero de 2021. 81 pacientes hipertensos atendidos por la clínica de salud familiar "Dr. Davi Marcos de Lima". Se invitó a los involucrados a responder un cuestionario que contenía datos sociodemográficos y la Escala de Disposición a Perdonar. Además, para el análisis estadístico se consideró un nivel de significancia del $95 \%$. Resultados: la muestra estuvo conformada por 81 individuos. En el presente estudio, hubo una prevalencia de mujeres $(66,7 \%)$, mayores o iguales a 60 años (63\%) y 53,7\% tenían Educación Primaria incompleta. Predominó el 50,6\% de los casados; El 66,7\% vive en pareja, el 29,6\% vive con una sola persona. La mayoría de las personas, el 69,1\%, no trabaja y / o está jubilado. La religión predominante fue la católica con un 74,1\%. Al correlacionar los datos sociodemográficos con la escala de perdón, las variables de estado civil y número de personas que conviven con hipertensos resultaron significativas (valor de $\mathrm{p}<0,005$ ). Conclusión: Con este estudio se puede ver que los pacientes hipertensos estaban dispuestos a perdonar. Además, el estudio mostró que el estado civil "casado" y "otros" interfieren con la voluntad del individuo de perdonar y que los hipertensos que viven con uno a tres individuos están dispuestos a perdonar más.

Palabras clave: Perdón; Hipertensión; Primeros auxilios; Enfermeras y enfermeras.

\section{Introdução}

Diversos estudos demonstram cada vez mais a estreita relação de doenças cardiovasculares com aspectos culturais, psicológicos e comportamentais. Nesse contexto, é possível observar que a implementação de estratégias de promoção de saúde tem papel fundamental na saúde de doentes, principalmente portadores de Doenças Crônicas Não Transmissíveis (DCNT) como a hipertensão arterial sistêmica (HAS). A HAS é compreendida como uma condição clínica multifatorial caracterizada por elevação sustentada dos níveis pressóricos $\geq 140$ e/ou 90 mmHg (Malachias et al., 2016).

No que tange o comportamento inclinado ao perdão correlacionado a HAS evidências afirmam que o perdão melhora o prognóstico de pacientes com essa patologia, já que praticar esse ato gera benefícios fisiológicos e redução de sentimentos negativos como o estresse e a raiva que fazem com que a pressão arterial se eleve (Pereira, 2013).

Observando alguns estudos correlatos ao exposto foi possível analisar que o perdão há algum tempo tem se tornado um pertinente objeto de pesquisa da Psicologia e demais áreas de conhecimento, sendo esse considerado como possível determinante de bem-estar, proporcionando diversas pesquisas em virtude de suas implicações na saúde mental e física dos seres humanos, principalmente embasados na perspectiva dos ofendidos (Gouveia et al., 2009; Pinho \& Falcone, 2015; Alencar \& Abreu, 2019).

Para Gouveia et al. (2015) o perdão é um desafio humano contínuo, pois, independente da condição em que foi ocasionado o dano ao indivíduo ofendido, ou seja, intencional ou involuntário, o ato de perdoar é uma atitude complexa. Pinho e Falcone (2018) identificam que a atitude em perdoar depende, sobretudo, da facilidade e propensão que o ofendido possui de perdoar algo ou alguém.

No entanto, para realizar o perdão, é necessário que a pessoa esteja disposta a concretizar tal ato, pois, a disposição para perdoar compreende um traço psicológico, individual e mutável que varia de acordo com as emoções, o ofensor e a capacidade que o indivíduo tem em perdoar (Gouveia et al., 2015). Ademais, o reconhecimento dos malefícios tanto pessoais 
quanto interpessoais que os sentimentos como mágoa, tristeza e rancor podem provocar ao indivíduo ofendido, é um dos fatores que interferem na disposição para perdoar (Pinho \& Falcone, 2018).

Baseado no conhecimento da interferência do perdão sobre a fisiologia do corpo humano surgiu uma reflexão problemática no que se refere a esse contexto. Diante disso, adveio o seguinte questionamento: qual o nível de disposição para perdoar em indivíduos com Hipertensão Arterial Sistêmica?

Apesar dos avanços e progressos técnico-científicos que promovem o desenvolvimento e o bem-estar humano, são poucas as pesquisas sobre o perdão e a disposição para perdoar e seus benefícios à saúde física e mental, consequentemente, a população em geral pouco sabe sobre a temática. No entanto, apesar disso, é evidente a relevância da temática à saúde pública, visto que a hipertensão é uma das principais de causas de morbimortalidade no mundo, diante disso, o estudo justifica-se em entender a relação entre a disposição ao perdão e a hipertensão, a fim de identificar os fatores predisponentes da hipertensão arterial sistêmica. Sendo assim, o presente estudo tem por objetivo, avaliar a disposição para perdoar em indivíduos com hipertensão arterial sistêmica.

\section{Metodologia}

Trata-se de um estudo transversal e quantitativo com abordagem descritiva e analítica (Köche, 2011) que envolveu 81 indivíduos com diagnóstico de Hipertensão Arterial Sistêmica que são assistidos pela Clínica de Saúde da Família Dr. Davi Marcos de Lima, no município de Lagarto (SE), entre março de 2020 e janeiro de 2021. A amostra se deu por conveniência e a coleta ocorreu nos domicílios dos participantes, sob mediação dos agentes comunitários de saúde, exceto um dia que aconteceu na Clínica de estudo. Os critérios de inclusão foram: ser atendido no Estabelecimento Assistencial de Saúde (EAS) acima referenciado e possuir hipertensão arterial sistêmica (HAS). Já nos critérios de exclusão estão os indivíduos que não são acompanhados por esta unidade de saúde, menores de 18 anos e pacientes que se recusaram a responder o questionário.

Para coleta de dados foi utilizado um instrumento contendo duas partes: a primeira compõe-se de um questionário elaborado pelas autoras com informações sociodemográficas: iniciais do nome; idade; sexo; raça/etnia; procedência; moradia; escolaridade; estado civil; situação conjugal; reside com; ocupação; religião e tempo de HAS. Já a segunda parte corresponde à Escala de Disposição para Perdoar (EDP). Foi elaborada por DeShea em 2003 (Deshea, 2003), no contexto estadunidense, em sua versão original intitulada de "A Willingness to Forgive (WTF)", composta inicialmente por 38 cenários, reduzidas a 19 cenários e atualmente, em sua versão final, contendo 12 cenários que oportuniza o participante a expressar sua resposta por meio de uma escala do tipo Likert cuja pontuação varia de 0 a 6 , sendo que 0 corresponde a nada disposto a perdoar e 6 totalmente disposto a perdoar. Para critérios de avalição do presente estudo, foi considerado que pontuações que variassem de 0 a 3 classificar-se-iam como nada ou pouco disposto a perdoar, já pontuações que variassem de 4 a 6 classificar-se-iam como muito ou totalmente disposto a perdoar (Gouveia et al., 2009). Essa escala foi validada no Brasil por Gouveia et al. (2015), em sua versão brasileira intitulada de "Escola de Disposição para Perdoar".

Nesse instrumento, os cenários utilizados contam com situações hipotéticas, como se tivessem acabados de ocorrer e não envolve pedidos de desculpa ou demonstração de arrependimento do ofensor. Essa maneira de avaliação da disposição de perdoar por meio da utilização de cenários é considerada como uma das melhores, sem que aja prejuízo aos participantes (Barbosa, 2015). É uma escala autoaplicável, e após a leitura o participante responde o quanto está disposto a perdoar o transgressor em cada situação. No entanto, como o público da pesquisa possui uma idade avançada e alguns não eram alfabetizados, as autoras liam o questionário para os participantes com essas limitações e eles respondiam.

Inicialmente, a coleta ocorreria nas mediações da unidade de saúde supracitada, durante os dias de atendimentos a doentes crônicos não transmissíveis. No entanto, em 11 de março de 2020 a Organização Mundial da Saúde decretou a pandemia de COVID-19, caracterizada como uma doença respiratória aguda, provocada pelo vírus SARS-CoV-2, tratava-se 
de uma nova cepa do coronavírus não detectada anteriormente em humanos, e foi identificado primeiramente como casos de pneumonia em dezembro de 2019 na cidade de Wuhan, na China (Organização Pan-Americana da Saúde/Organização Mundial da Saúde [OPAS/OMS], 2020). Com o estabelecimento da pandemia e a identificação dos primeiros casos de Covid19 no Brasil, os números de atendimentos de doentes crônicos não transmissíveis nos estabelecimentos de saúdes no Brasil, reduziram significantemente, incluindo os atendimentos na Clínica de Saúde da Família Dr. Davi Marcos de Lima.

Considerando esse contexto, tornou-se necessário uma mudança na forma de coleta dos dados para a pesquisa. A alternativa encontrada foi realizar a coleta nos domicílios dos hipertensos. Para isso, as pesquisadoras contataram os enfermeiros, e estes, permitiram que os Agentes Comunitários de Saúde (ACS) mediassem a coleta. Em seguida, as pesquisadoras entraram em contato com os ACS e foram marcando gradativamente os dias para as coletas. Mediante acordo, os ACS e as pesquisadoras encontravam-se na data, dia e local combinados. Os ACS e as pesquisadoras percorriam a respectiva microárea de responsabilidade do ACS. Como os ACS conheciam os hipertensos e seus respectivos domicílios, eles guiavam-nas até as residências onde se encontravam os pacientes que, baseados nos critérios de inclusão e exclusão, incluíamse nas pesquisas.

A coleta ocorreu seguindo os parâmetros de segurança recomendados pela Organização Mundial da Saúde (OMS), respeitando medidas de distanciamento, e fazendo utilização de máscaras e álcool em gel, vale ressaltar que as pesquisadoras não entraram nas residências, limitando-se aos espaços da garagem e varandas dos domicílios dos participantes. As autoras convidaram os hipertensos a participar da pesquisa, explicando objetivos, riscos e benefícios envolvidos.

Os dados do questionário de caracterização dos participantes, bem como os dados referentes a Escala de Disposição para Perdoar (EDP) foram tabulados no Microsoft Software Excel. As variáveis categóricas foram descritas por meio de frequência absoluta e relativa percentual. As variáveis contínuas foram descritas por meio de média, mediana, desvio padrão e intervalo interquartil. A hipótese de normalidade intragrupo foi testada por meio do teste de Shapiro-Wilks. Quando confirmada as diferenças de média foram testadas por meio dos testes T para amostras independentes ( 2 grupos) ou ANOVA ( 3 ou mais grupos), caso contrário, foi testada por meio dos testes Mann-Whitney (2 grupos) ou Kruskal-Wallis (3 ou mais grupos). As correlações entre variáveis contínuas foram testadas por meio das correlações de Pearson e Spearman. O nível de significância adotado foi de 5\% e o software adotado foi o R Core Team 2021.

Mediante o esclarecimento dos objetivos da pesquisa, seus potenciais benefícios, ausência de danos e que seria assegurada a confidencialidade, privacidade das informações, foi apresentado o Termo de Consentimento Livre e Esclarecido, assim como o instrumento de coleta de dados, quando aceitaram participar da pesquisa. Cumprindo os princípios do rigor ético da Resolução 466/12 (Brasil, 2013a). O estudo foi aprovado pelo Comitê de Ética em Pesquisa com Seres Humanos (CEP) CAAE (01922918.6.0000.5546) e sob o parecer n $n^{\circ}$ 3.013.686. Além disso, a pesquisa seguiu o que compete a resolução ética $\mathrm{n}^{\circ} 510$ de 7 de abril de 2016 (Brasil, 2016).

\section{Resultados}

O estudo realizado compôs-se de 81 pacientes, dos quais, 54 (66,7\%) eram do sexo feminino e 27 (33,3\%) do sexo masculino. Em relação à idade, houve uma variação de indivíduos entre 32 e 89 anos, sendo que 51 (63\%) correspondia a faixa etária $\geq 60$ anos. Houve predominância da cor parda 41 (50,6\%), da procedência zona urbana 78 (96,3\%) e da moradia própria, 71 (87,7\%). Quanto à escolaridade, 43 (53,1\%) possuíam ensino fundamental incompleto. Em relação ao estado civil, houve predominância de indivíduos casados 41 (50,6\%), além disso, 54 (66,7\%) vivem com o companheiro. Sequencialmente, quando questionado com quantas pessoas o entrevistado reside, foi constatado que 24 (29,6\%) residem apenas com uma pessoa, seguido de $21(26,0 \%)$ residem com mais três pessoas, além dela. No tocante à ocupação dos envolvidos, 56 (69,1\%) não trabalham e/ou estão aposentados. A religião predominante foi católica com 60 (74,1\%). Por fim, quanto aos anos de 
hipertensão $58(71,6 \%)$ afirmou possuir mais de 5 anos com o diagnóstico. Na tabela abaixo, encontram-se dispostos o detalhamento dos dados sociodemográficos obtidos.

Tabela 1 - Dados sociodemográficos de pacientes hipertensos, Lagarto/SE, Brasil, 2021.

\begin{tabular}{|c|c|c|c|c|c|c|}
\hline & $\mathbf{N}$ & $\%$ & Média & DP & Mediana & IIQ \\
\hline Idade & & & 63,62 & 12,79 & 63 & $55-71$ \\
\hline \multicolumn{7}{|l|}{ Faixa Etária } \\
\hline $32-44$ & 5 & 6,1 & & & & \\
\hline $45-59$ & 25 & 30,9 & & & & \\
\hline$>=60$ & 51 & 63,0 & & & & \\
\hline \multicolumn{7}{|l|}{ Sexo } \\
\hline Feminino & 54 & 66,7 & & & & \\
\hline Masculino & 27 & 33,3 & & & & \\
\hline \multicolumn{7}{|l|}{ Raça/Etnia } \\
\hline Branca & 21 & 25,9 & & & & \\
\hline Preta & 19 & 23,5 & & & & \\
\hline Parda & 41 & 50,6 & & & & \\
\hline \multicolumn{7}{|l|}{ Procedência } \\
\hline Rural & 3 & 3,7 & & & & \\
\hline Urbana & 78 & 96,3 & & & & \\
\hline \multicolumn{7}{|l|}{ Moradia } \\
\hline Própria & 71 & 87,7 & & & & \\
\hline Alugada & 6 & 7,4 & & & & \\
\hline Cedida & 4 & 4,9 & & & & \\
\hline \multicolumn{7}{|l|}{ Escolaridade } \\
\hline Não Estudou & 19 & 23,5 & & & & \\
\hline Fundamental Completo & 2 & 2,5 & & & & \\
\hline Fundamental Incompleto & 43 & 53,1 & & & & \\
\hline Ensino Médio Completo & 6 & 7,4 & & & & \\
\hline Ensino Médio Incompleto & 7 & 8,6 & & & & \\
\hline Ensino Superior & 4 & 4,9 & & & & \\
\hline \multicolumn{7}{|l|}{ Estado Civil } \\
\hline Solteiro & 13 & 16,0 & & & & \\
\hline Casado & 41 & 50,6 & & & & \\
\hline União Estável & 12 & 14,9 & & & & \\
\hline Outro & 15 & 18,5 & & & & \\
\hline \multicolumn{7}{|l|}{ Situação Conjugal } \\
\hline Vive com companheiro & 54 & 66,7 & & & & \\
\hline Vive sem companheiro & 27 & 33,3 & & & & \\
\hline \multicolumn{7}{|l|}{ Reside com } \\
\hline Sozinho & 7 & 8,6 & & & & \\
\hline Uma pessoa & 24 & 29,6 & & & & \\
\hline Duas pessoas & 17 & 21,0 & & & & \\
\hline Três pessoas & 21 & 26,0 & & & & \\
\hline Quatro pessoas ou mais & 12 & 14,8 & & & & \\
\hline \multicolumn{7}{|l|}{ Ocupação } \\
\hline Autônomo & 11 & 13,6 & & & & \\
\hline Servidor público & 3 & 3,7 & & & & \\
\hline Carteira assinada & 1 & 1,2 & & & & \\
\hline Não trabalha/aposentado & 56 & 69,1 & & & & \\
\hline Outro & 10 & 12,3 & & & & \\
\hline \multicolumn{7}{|l|}{ Religião } \\
\hline Católico & 60 & 74,1 & & & & \\
\hline Evangélico & 12 & 14,9 & & & & \\
\hline Adventista & 4 & 4,9 & & & & \\
\hline Sem religião & 4 & 4,9 & & & & \\
\hline Outro & 1 & 1,2 & & & & \\
\hline \multicolumn{7}{|l|}{ Anos de Hipertensão } \\
\hline Menos de 1 ano & 1 & 1,2 & & & & \\
\hline Entre 1 e 5 anos & 22 & 27,2 & & & & \\
\hline Mais de cinco anos & 58 & 71,6 & & & & \\
\hline EDP & & & 43,49 & 17,46 & 42 & $31-58$ \\
\hline
\end{tabular}


Legenda: $\mathrm{n}$ - frequência absoluta. \% - frequência relativa percentual. DP - Desvio Padrão. IIQ - Intervalo interquartil. EDP Escala de Disposição para Perdoar. Fonte: Autores (2021).

Considerando os 81 participantes da pesquisa e os 12 cenários que compõem a EDP, obteve-se um total de 972 respostas, distribuídas nas pontuações que variam de 0 a 6 . No tocante a isso, observou-se que 162 respostas na pontuação “ 0 ”, 54 respostas pontuaram "1”, 91 respostas “2”, 129 respostas pontuaram “3”, 95 respostas "4”, 126 respostas na pontuação "5" e 315 pontuaram o número "6". Sendo assim, obteve-se um total de 436 respostas, considerados como pouco ou nada disposto a perdoar, enquanto que 536 respostas pontuaram como muito ou totalmente disposto a perdoar. No gráfico abaixo encontramse dispostos os dados supracitados.

Gráfico 1 - Distribuição das respostas da Escala de Disposição para Perdoar, Lagarto, Sergipe, 2021.

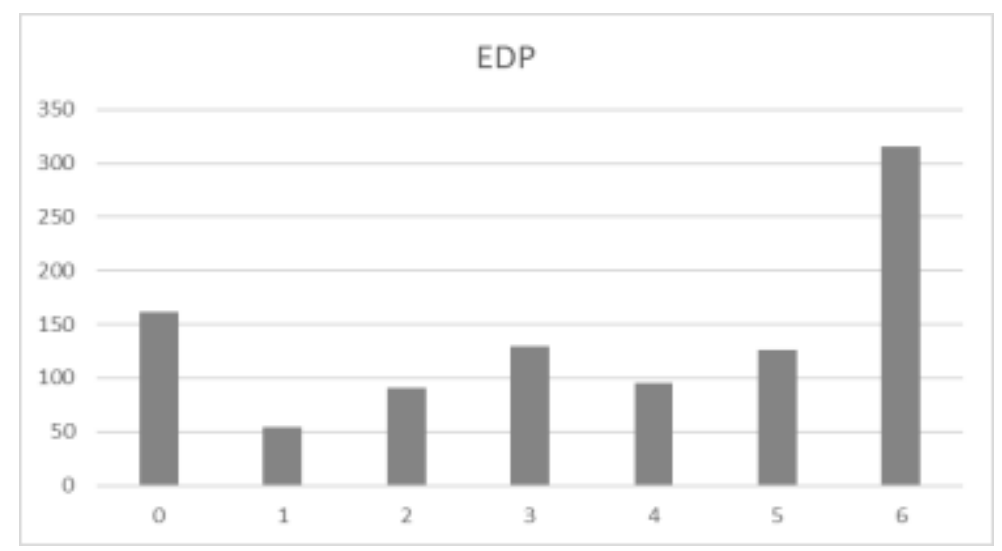

Fonte: Autores (2021).

Correlacionando a Escala de Disposição para Perdoar (EDP) com o perfil dos hipertensos, foram encontradas duas situações com significância estatística, considerando p-valor <0,05. Pode-se destacar que o estado civil (p-valor <0,001) pode interferir na disposição que a pessoa tem a perdoar, observando que indivíduos casados possuem mais propensão ao perdão, sendo que a média obtida foi de 41,4 (DP 16,3) e mediana de 39, do que os indivíduos em União estável com média de 36,6 (DP 18,7) e mediana de 30 (24,3-45,8). Além disso, outro resultado constatado foi que os participantes que residem com uma pessoa ou até três pessoas (p-valor <0,001) são mais propensas a perdoar, sendo que na variável de indivíduos que moram sozinhas com média de 52,9 (DP 21,5) e mediana de 64 (33-67) são mais propensas a perdoar do que os indivíduos que moram com quatro pessoas ou mais com média de 37,1 (DP 16,4) e mediana de $34(25,3-42,5)$. A Tabela 2, a seguir, apresenta o detalhamento dos dados: 
Research, Society and Development, v. 10, n. 10, e585101019174, 2021

(CC BY 4.0) | ISSN 2525-3409 | DOI: http://dx.doi.org/10.33448/rsd-v10i10.19174

Tabela 2 - Correlação entre os dados sociodemográficos e a disposição para perdoar, Lagarto. Sergipe, 2021.

\begin{tabular}{|c|c|c|c|}
\hline & Média (DP) & Mediana (IIQ) & p-valor \\
\hline \multicolumn{4}{|l|}{ Faixa Etária } \\
\hline $32-44$ & $33,8(12,1)$ & $39(21-44)$ & $0,103^{\mathrm{A}}$ \\
\hline $45-59$ & $49,0(15,8)$ & $51(36-64)$ & \\
\hline$>=60$ & $41,2(18,1)$ & $41(30-56)$ & \\
\hline \multicolumn{4}{|l|}{ Sexo } \\
\hline Feminino & $44,1(16,9)$ & $43(33,3-59,3)$ & $0,675^{\mathrm{T}}$ \\
\hline Masculino & $42,3(18,8)$ & $38(27-56)$ & \\
\hline \multicolumn{4}{|l|}{ Raça/Etnia } \\
\hline Branca & $41(19,3)$ & $38(25,5-58)$ & $0,326^{\mathrm{K}}$ \\
\hline Preta & $48,4(15)$ & $48(38-63)$ & \\
\hline Parda & $42,5(17,5)$ & $42(28-58)$ & \\
\hline \multicolumn{4}{|l|}{ Procedência } \\
\hline Rural & $50(19)$ & $45(34-0)$ & $0,610^{\mathrm{M}}$ \\
\hline Urbana & $43,2(17,5)$ & $42(30,8-57,5)$ & \\
\hline \multicolumn{4}{|l|}{ Moradia } \\
\hline Própria & $43,6(18,2)$ & $43(30-60)$ & $0,991^{\mathrm{K}}$ \\
\hline Alugada & $43,2(14,3)$ & $41,5(33-54)$ & \\
\hline Cedida & $42,8(8,2)$ & $39(38-51,3)$ & \\
\hline \multicolumn{4}{|l|}{ Escolaridade } \\
\hline Não Estudou & $47,4(15,6)$ & $48(37-57)$ & $0,429 \mathrm{~K}$ \\
\hline Fundamental Completo & $49,5(17,7)$ & $49,5(37-0)$ & \\
\hline Fundamental Incompleto & $41,7(18,6)$ & $43(25-59)$ & \\
\hline Ensino Médio Completo & $52(14,3)$ & $49(38,8-66,8)$ & \\
\hline Ensino Médio Incompleto & $34,6(18,5)$ & $31(18-41)$ & \\
\hline Ensino Superior & $43,8(14,3)$ & $44(29,8-57,5)$ & \\
\hline \multicolumn{4}{|l|}{ Estado Civil } \\
\hline Solteiro ${ }^{b}$ & $52,6(16,3)$ & $54(39-69,5)$ & $<0,001^{A}$ \\
\hline Casado ${ }^{\text {a,b }}$ & $41,4(16,3)$ & $39(32,5-55,5)$ & \\
\hline União Estável ${ }^{\text {b }}$ & $36,6(18,7)$ & $30(24,3-45,8)$ & \\
\hline Outro ${ }^{a, b}$ & $46,7(18,3)$ & $43(38-64)$ & \\
\hline \multicolumn{4}{|l|}{ Situação Conjugal } \\
\hline Vive Com companheiro & $42(17)$ & $40,5(29,8-56)$ & $0,279^{\mathrm{T}}$ \\
\hline Vive Sem companheiro & $46,5(18,4)$ & $43(34-64)$ & \\
\hline \multicolumn{4}{|l|}{ Reside com } \\
\hline Sozinho b & $52,9(21,5)$ & $64(33-67)$ & $<0,001^{\mathrm{A}}$ \\
\hline Uma pessoa $^{\mathrm{a}, \mathrm{b}}$ & $43(17,7)$ & $41,5(30,3-57,8)$ & \\
\hline Duas pessoas ${ }^{\mathrm{a}, \mathrm{b}}$ & $42,5(15,5)$ & $43(32-55,5)$ & \\
\hline Três pessoas a,b & $45,5(17,9)$ & $45(35,5-58,5)$ & \\
\hline Quatro pessoas ou mais ${ }^{\text {a }}$ & $37,1(16,4)$ & $34(25,3-42,5)$ & \\
\hline \multicolumn{4}{|l|}{ Ocupação } \\
\hline Autônomo & $49,2(20,6)$ & $55(37-65)$ & $0,661^{\mathrm{K}}$ \\
\hline Servidor público & $34,3(6,4)$ & $38(27-0)$ & \\
\hline Não trabalha/aposentado & $43(17,3)$ & $42,5(31-56)$ & \\
\hline Outro & $43,4(18,4)$ & $42,5(28,5-61,3)$ & \\
\hline \multicolumn{4}{|l|}{ Religião } \\
\hline Católico & $40,5(16,7)$ & $38(27,5-53,5)$ & $0,054^{\mathrm{K}}$ \\
\hline Evangélico & $54,5(16,2)$ & $56,5(45,5-69,5)$ & \\
\hline Adventista & $47,3(22,6)$ & $53(23,5-65,3)$ & \\
\hline Sem religião & $46(17)$ & $39,5(34,8-63,8)$ & \\
\hline \multicolumn{4}{|l|}{ Anos de Hipertensão } \\
\hline Entre 1 e 5 anos & $49,7(14)$ & $51,5(36,5-62,3)$ & $0,051^{\mathrm{M}}$ \\
\hline \multirow[t]{2}{*}{ Mais de cinco anos } & $40,9(18,2)$ & $39(26,8-54,3)$ & \\
\hline & $\mathrm{P}(\mathrm{p}$-valor) & $\mathrm{R}$ (p-valor) & \\
\hline Idade & $-0,025(0,827)$ & $-0,064(0,569)$ & \\
\hline
\end{tabular}

Legenda: DP - Desvio Padrão. IIQ - Intervalo interquartil. T - Teste T para amostras independentes. M - Teste de Mann-Whitney. A - ANOVA. K - Teste de Kruskal-Wallis. a,b Subgrupos distintos ao nível de 5\% para o teste de Duncan. P - Correlação Linear de Pearson. R - Correlação de Spearman. Fonte: Autores (2021).

\section{Discussão}

Embora não exista na literatura um consenso entre os autores sobre o conceito de perdão, a disposição para perdoar e a decisão do perdão tem se tornado objetos crescentes de interesse e pesquisas nas diversas áreas de conhecimento, visto que, 
estudos têm revelado frequentemente a associação do perdão com benefícios fisiológicos, psicológicos, sociais e evolutivos (Santana \& Lopes, 2012; Gouveia et al., 2009; Pinho \& Falcone, 2015; Alencar \& Abreu, 2019). Para tanto, o ato de perdoar engloba mecanismos neurocognitivos e afetivos, e, além disso, demonstra ser um fator importante de mudança de comportamento, principalmente envolvendo a interação entre pares (Santana, 2011).

Estudos comprovam que o perdão ou até mesmo a disposição para perdoar causa melhora no prognóstico em DCNT e em doenças coronárias, ou seja, um indivíduo com mais disposição ao perdão tende a ter menos complicações do que aqueles que se predispõe menos (Salmazi, 2015). Sabe-se que a ausência do perdão causa estresse e em consequência disso há alterações fisiológicas como elevação da respiração, pressão arterial e frequência cardíaca (Worthington \& Scherer, 2004). Além disso, a prática do perdão está relacionada a menor pressão arterial diastólica e a redução da probabilidade de acontecimentos de hipertensão arterial (Barroso et al., 2021).

Dados mundiais revelam que a Hipertensão Arterial Sistêmica afeta cerca de $20-40 \%$ da população adulta (OPAS/OMS, 2016), sendo que há uma discreta diferença de prevalência de HAS entre os gêneros, possivelmente pela predominância em homens mais jovens, até os 50 anos, e em mulheres mais idosas, após a $5^{\circ}$ década de vida (Hajjar, Kotchen $\&$ Kotchen, 2006). No presente estudo obteve-se uma prevalência do sexo feminino. Índices semelhantes foram encontrados em um estudo envolvendo hipertensos no município de Viçosa-MG, revelando predominância do sexo feminino, 74,1\% (Mendonça et al., 2015). Assim como observados nas investigações realizadas com indivíduos hipertensos dos municípios de Diamantina-MG e Aracaju-SE, com frequência de 56,9\% e 75,9\%, respectivamente (Ferreira, Bodevan \& Oliveira, 2019; Gois et al., 2016).

Além desses estudos, dados da Vigilância de Fatores de Risco e Proteção para Doenças Crônicas por Inquérito Telefônico (Vigitel) de 2018 (Brasil, 2019), evidenciaram que a prevalência de HAS nas capitais brasileiras era de 24,7\%, sendo que a prevalência foi maior entre as mulheres $(27,0 \%)$ do que entre os homens $(22,1 \%)$. Esse predomínio é esclarecido devido à maior percepção das mulheres sobre sua condição de saúde, mais conhecimento das mulheres a respeito do diagnóstico da HAS e maior busca aos serviços de saúde (Cesarino, 2008; Zaitune, Barros, César, Carandina \& Goldbaum, 2006). Considerando que os ACS que foram responsáveis pela mediação entre os hipertensos e as pesquisadoras, a prevalência feminina pode-se dar também em decorrência do melhor relacionamento entre os ACS e as mulheres hipertensas, visto que essas tendem a ser mais receptivas e preocupadas com os problemas de saúde.

Os resultados supracitados podem estar associados também ao uso prévio de contraceptivos orais pelas mulheres durante a idade fértil. Uma meta-análise envolvendo 24 estudos evidenciou que o uso de anticoncepcionais orais aumenta o risco de hipertensão (Liu, 2017). Essa elevação da PA ocorre duas vezes mais em mulheres usuárias dos contraceptivos orais, e pode haver um aumento em 4 a $9 \mathrm{mmHg}$ do nível basal. Nas mulheres, soma-se a faixa etária elevada, alterações hormonais e metabólicas decorrentes da menopausa, já que há um aumento da prevalência de HAS em mulheres durante a menopausa (Barbosa, Guimarães \& Saraiva, 2008).

Já em uma pesquisa de Silva (2020) que associa dados sociodemográficos com o perdão, foi evidenciado que homens e mulheres perdoam em um nível significativamente equivalentes, porém foi demonstrado que as mulheres tendem a ser mais resolutivas a partir da consciência do ofensor sobre o ocorrido. Ademais, na pesquisa de Fonseca et al. (2017), foi identificado que o homem é mais benevolente e as mulheres tem maior índices de emitir sentimentos como ressentimento e evitação. Em contrapartida, foi possível observar pela fala de alguns autores que as mulheres conseguem perdoar com mais facilidade, pois estas preferem manter um ambiente e relação de harmonia quando comparada aos homens, já que estes tendem a ser mais vingativos e agressivos (McCullough, Bellah, Kilpatrick \& Johnson, 2001).

Percebe-se que esse estudo obteve uma prevalência de indivíduos com muita ou total disposição ao perdão, e tais resultados podem estar associados a idade prevalente dos participantes, já que indivíduos mais velhos tendem a perdoar mais. 
A presente análise corrobora com os achados de Fonsêca et al. (2017), já que não foi encontrado associação entre perdão e idade. Não obstante, no que discerne o estudo de Gouveia et al. (2009) dentre os dados sociodemográficos utilizados na pesquisa, a idade foi a única variável em que se apresentou importante na disposição para perdoar, justificando-o que a idade pode significar maturidade do pensamento na compreensão das situações. Assim como, o estudo de Silva (2020) que identificou que a maior idade possui maior predisposição a perdoar seus ofensores, independentemente do ocorrido.

Sendo assim, com o passar dos anos, as pessoas conseguem realizar o ato de perdoar com mais facilidade quando comparado com os mais jovens, decorrente da vivência de transgressões ocorridas ao longo da vida, dando maior ênfase a aspectos emocionais que consequentemente facilitará o perdão, além disso, a faixa etária maior tende a buscar manter as relações mais saudáveis, enquanto os jovens tendem a ser mais vingativos, trazendo sentimentos negativos como a raiva, rancor e mágoa e consequentemente alterações fisiológicas como a elevação da pressão arterial, aumento da frequência cardíaca e da frequência respiratória, além disso, esses indivíduos submetem-se ao maior risco de desenvolver doenças cardiovasculares, renais, cerebrais e crônicas, bem como, há o aumento da probabilidade do desenvolvimento de distúrbios de ansiedade e depressão. (Worthington \& Scherer, 2004; Allemand, 2008; Ghaemmaghami, Allemand \& Martin, 2011; Steiner, Allemand \& McCullough et al., 2001; Silva, 2020).

Nesse sentido, percebe-se que há o aumento da disposição ao perdão ao longo da vida dos indivíduos, no entanto, concomitante a isso, sabe-se que a prevalência de HAS também aumenta com a idade, corroborando com os dados encontrados nesse estudo. Observa-se que os indivíduos tendem a perdoar mais com o aumento da idade devido as experiências vividas, bem como, ao aprender com as diversas situações estressoras da vida, estas situações caracterizam-se como fatores predisponentes a doenças crônicas não transmissíveis, e esse acúmulo de experiências negativas ao longo da vida pode resultar posteriormente em aumento da probabilidade de ocorrência de hipertensão arterial. De modo geral, o estudo de Ferreira et al. (2019), apresentou prevalência de indivíduos com HAS com idade superior ou igual a 45 anos. Já em outro estudo na cidade de São José do Rio Preto a prevalência de idade foi de 53,8 anos (Cesarino et al., 2008). Além disso, Gois et al. (2016) também evidenciam em sua pesquisa que há predominância da faixa etária acima de 60 anos.

Contudo, sabe-se que esses valores podem variar de acordo com a população abordada para os estudos. Segundo a VI Diretrizes Brasileiras de Hipertensão, 7,6 milhões de pessoas morrem por ano devido à hipertensão e dentre os fatores causadores desta morte estão os que possuem idade entre 45 e 69 anos (Sociedade Brasileira de Cardiologia [SBC], 2010). Outro ponto a ser considerado é que a idade se apresenta como um forte fator de risco para a HAS, ademais, essa DCNT pode ser desencadeada por fatores modificáveis como estilo de vida ou por genética, que é um fator de risco não modificável (Brasil, 2013b).

Como visto, houve prevalência de idade superior a 60 anos, este resultado associa-se com os dados encontrados na variável ocupação. Observa-se uma prevalência semelhante de aposentados, 58\%, em indivíduos com HAS em dados encontrados na pesquisa de Mendonça et al. (2015) e encontrados em Gois et al. (2016), 62,7\%. Dados encontrados em um estudo envolvendo hipertensos de um município do noroeste do Paraná evidenciaram também a prevalência de indivíduos aposentados/pensionistas, 55,2\% (Lucena et al., 2021).

Essa prevalência de indivíduos hipertensos aposentados/pensionistas associa-se, sobretudo, a idade, já que evidências revelam que a o aumento da idade, relaciona-se diretamente com o diagnóstico de hipertensão, pois além de uma vida submetida a fatores estressores, má alimentação e falta de atividade física, o aumento da idade relaciona-se com alterações fisiológicas e físicas, como enrijecimento das paredes do vaso sanguíneo e consequentemente com a elevação da pressão arterial. Além disso, pessoas aposentadas, que normalmente aposentam-se por idade ou por invalidez, tornam-se ou mantém-se sedentários, fator esse, que predispõe o surgimento de HAS. 
Quanto à raça/etnia, a maioria declarou-se da cor parda, porém quando se analisa estudos sobre etnia dos hipertensos, é possível observar que há uma forte predominância da HAS em negros e indígenas podendo ser caracterizada por fatores étnicos ou socioeconômicos (Brasil, 2012a; Brasil, 2013b). Porém, diferentemente do estudo anterior, para Toledo et al. (2020), há uma maior vulnerabilidade para desenvolver tal patologia pessoas que se declaram pardas e negras, não havendo diferença entre as duas etnias/raças.

Segundo a Sociedade Brasileira de Cardiologia (2010) a HAS é duas vezes mais prevalente em indivíduos não brancos. Em concordância com essa e outras pesquisas, foi observado que usaram os termos 'brancos' e 'não brancos' para obter estes dados, neles, há um maior número de pessoas com HAS apresentando etnia não branca (Zaitune et al., 2006; Basset Jr, Fitzhugh, Crespo, King \& McLaughlin, 2002). Estudos acreditam que a prevalência de HAS em pessoas não brancas, estejam ligadas a fatores genéticos, no entanto, não há, até o momento, estudos que ratifiquem a hipótese e que evidenciem a predisposição que o indivíduo tem em desenvolver a HAS (SBC, 2010). Além disso, outros aspectos podem ser contribuintes dessas disparidades, como situação social e econômica, hábitos de vida e acesso aos serviços de saúde (Zaitune et al., 2006).

Apesar de não ter encontrado na literatura associação entre tipo de procedência e tipo de moradia e hipertensão arterial, a prevalência de procedência em área urbana, dá-se devido a área de abrangência da Clínica de Saúde da Família Dr. Marcos de Lima, na qual assiste em maior extensão uma área adscrita à zona urbana.

No que tange a escolaridade, o maior número identificado no estudo foi de pessoas com HAS com ensino fundamental incompleto. O nível de escolaridade está relacionado com o perfil socioeconômico de cada indivíduo. Este, desempenha papel fundamental nas condições de saúde em decorrência do acesso ao sistema de saúde, grau de informação, compreensão do problema e adesão ao tratamento (Ong, Cheung, Man, Lau \& Lam, 2007). Ong et al. (2007) expõem que quanto menor a escolaridade, maior desenvolvimento de HAS quando comparado com pessoas com maior grau de instrução, fato também encontrado em outros estudos (Zaitune et al., 2006; Mendonça et al., 2015; Ferreira et al., 2019). Esta situação pode ocorrer devido ao trabalho que o indivíduo exerce, podendo ser mais pesado e consequentemente mais estressante. Para tanto, seriam necessários mais estudos a respeito do tema para confirmação da tese (Ferreira et al., 2019).

Em relação ao estado civil houve predomínio de indivíduos casados que conviviam com companheiro. Dados semelhantes foram encontrados no estudo de Cesarino et al (2008) revelando que 63,9\% dos hipertensos eram casados e no estudo envolvendo hipertensos de Alfena- MG, em que 60\% dos hipertensos atendidos no Centro de Atendimentos às Doenças Crônicas em 2015 eram casados (Silva et al., 2016). Um estudo realizado por Gois et al. (2016) expôs que 50,2\% dos indivíduos com HAS viviam com o companheiro.

Tais dados encontrados corroboram com a caracterização sociodemográfica dos hipertensos de Viçosa-MG, que revelam que há uma prevalência de indivíduos com HAS que vivem acompanhados seja do companheiro e/ou filhos (Mendonça et al., 2015). Para tanto, uma revisão de literatura brasileira contendo dez estudos, identificou que houve uma prevalência de HAS entre indivíduos casados. Esses resultados podem estar relacionados a maior responsabilidade familiar (Cesarino, 2008).

A prevalência de HAS em indivíduos casados e que vivem pelo menos com uma pessoa no estudo, associa-se à maior responsabilidade dos indivíduos, tanto responsabilidades domésticas, quanto profissionais. Além disso, relaciona-se a maior chance de conflitos que pode haver, quando se vive com outras pessoas, tais conflitos geram estresses, raiva, mágoa e que com a falta do perdão representa um aspecto que interfere na manutenção da pressão arterial.

Por sua vez, o resultado do presente estudo está em concordância com o pensamento de McCullough e Witvliet (2001), pois estes definem o perdão em três perspectivas, sendo que a terceira corresponde às unidades sociais, nesse sentido, associa o perdão com uma habilidade de confiança e intimidade e ainda, caracteriza que algumas estruturas sociais estimulam um maior grau de perdão, como casamento, família e comunidade, e outras menor grau de perdão. Para Bueno, Souza, 
Monteiro e Teixeira (2013) o casamento refere-se a uma estrutura adulta que envolve simultaneamente a individualidade e o pertencimento por meio de uma fusão-individuação.

Sabe-se que conflitos e ofensas ocorrem comumente em indivíduos envolvidos em relacionamentos, sobretudo, íntimos, já que ofensas provocadas por estranhos tendem a possuir menor carga afetiva (Andrade, 2014). Em um estudo envolvendo um relato de 35 ofensas, constatou-se que todas ocorreram com pessoas do convívio diário, sendo que 25,7 \% foram com colegas de trabalho, 22,8\% com os pais, $22,8 \%$ com cônjuges, $20 \%$ com outros familiares e $8,7 \%$ com amigos (Pinho \& Falcone, 2018). Nesse sentido, vê-se a importância da prática do perdão no casamento, visto que a convivência diária, somadas a ideias e pensamentos diferentes podem gerar ou agravar conflitos.

Para Worthington et al. (2015), a vivência do perdão no início da vida matrimonial, principalmente, se os recémcasados adquirem a prática do diálogo e da reconciliação, certamente pode ser uma experiência positiva e enriquecedora para prevenção e gestão de conflitos futuros. Assim como o estudo de Pinho e Falcone (2018) identificou, dentre outros fatores, o diálogo esclarecedor como facilitador do perdão, assemelhando-se ao pensamento de Worthington et al. (2015).

Alguns autores caracterizam o perdão como um ato extraordinário, no entanto, McCullough et al. (2001), acreditam que o perdão é dificilmente concedido quando envolve ofensas intensas, diferentemente de desapontamentos corriqueiros causados frequentemente durante a convivência, esse tipo de perdão é denominado de ordinário e as experiências vivenciadas tanto da ofensa quanto do ato de perdoar são menos profundas (McCullough et al., 2001). Tais desentendimentos comuns ocorrem entre casais e consequentemente o perdão é concedido. McCullought et al. (2001) refere ainda que pessoas que vivenciaram experiências extraordinárias têm mais probabilidade de conceder o perdão em situações menores, em contrapartida, a vivência frequente de ofensas ordinárias pode resultar em acúmulos de trauma e consequentemente em uma experiência extraordinária.

A correlação significativa entre a quantidade de indivíduos residentes com os hipertensos está associada tanto com o estado civil, visto que a maioria dos hipertensos envolvidos é casada, como está relacionada a outros fatores, sobretudo, de relação de confiança e intimidade como exposto anteriormente. Em um estudo realizado para avaliar disposição para o perdão em pacientes acometidos com infarto agudo do miocárdio em comparação com indivíduos que não possuem doença coronária crônica, foi identificado que a maioria dos envolvidos residia com uma ou até três pessoas, no entanto, não houve diferença significativa entre os grupos (Avezum, 2018).

Sabe-se que indivíduos que residem em um mesmo lar tendem a possuir relações íntimas, harmoniosas e afetivas, isso ocorre em consequência da aproximação efetiva entre os membros. Dessa forma, assim como nas relações conjugais, existe a possibilidade de haver desentendimentos decorrentes de visões e opiniões de mundo diferente, gostos e escolhas distintas, dependência financeira ou até mesmo dependência maternal e patriarcal. Diante desse cenário de convívio diário e da estreita relação afetiva entre tais relacionamentos, observa-se que quanto mais próximo é o ofensor, maior e mais dolorosa é a ofensa, podendo provocar decepção, quebra de confiança e sentimento de traição no ofendido (Silva, 2020).

Quanto à religiosidade os dados encontrados foram semelhantes aos vistos nas literaturas disponíveis. Tais dados foram equivalentes aos encontrados na pesquisa de Mendonça et al. (2015), tendo revelado 79,7\% católicos e 19,6\% evangélicos/protestantes. Estes achados corroboram com os apresentados por Gois et al. (2016), em que 78,9\% possuem alguma religião. Mediante ao predomínio no estudo de indivíduos que possuíam alguma religião, era esperado tanto para os autores (Gouveia et al., 2009) quanto para as pesquisadoras do referido estudo era que quanto maior o nível de religiosidade dos indivíduos, maior a propensão do perdão (Gouveia et al., 2009; Fonsêca et al., 2017).

Estudos desenvolvidos por Tsang, McCullough e Hoyt (2005) demonstram que a religiosidade está sim diretamente ligada com o perdão. Isso pode ser justificado devido ao vínculo com a religiosidade e consequentemente com Deus, faz com que as pessoas que seguem alguma religião consigam perdoar com mais facilidade além de possuírem uma visão específica de 
certas situações podendo estar ligada a empatia e compaixão (Rye et al., 2001). Certamente, existe na literatura uma divergência entre a associação de perdão e religião, podendo isso estar relacionado a uma falta de escala que avalie adequadamente essa variável (Gouveia et al., 2009). Além disso, essa discrepância é resultado de uma falta de diferenciação entre os conceitos de "religião", "espiritualidade" e "religiosidade". No presente estudo, foi questionado apenas se o indivíduo possui religião e se possui, qual seria a religião, no entanto, não foram identificados aspectos de religiosidade e espiritualidade. Um estudo semelhante utilizando a EDP foi realizado para avaliar a disposição ao perdão e infarto agudo do miocárdio (IAM) e sua relação com espiritualidade e religiosidade, os dados sugeriram que pessoas com IAM possuíam menos disposição para perdoar em algumas das situações avaliadas (Avezum, 2018).

No que concerne ao tempo de diagnóstico da HAS, os achados assemelham-se com os dados encontrados em uma pesquisa realizada com hipertensos do município de Recife-PE, em que 51,7\% dos hipertensos sabiam do diagnóstico há mais de dez anos e 21,7\%, entre 6 e 10 anos. Já o estudo realizado com hipertensos de Alfenas-MG revelou que 43,2\% possuem diagnóstico há mais de 39 anos, sendo uma média de 15 anos (Silva et al., 2016). Essa prevalência de média alta do tempo de diagnóstico da HAS está associada provavelmente a identificação oportuna do diagnóstico de HAS.

O enfermeiro da atenção básica tem importante papel na identificação das alterações dos níveis pressóricos dos pacientes, pois a HAS caracteriza- se como uma doença crônica não transmissível e em muitos casos, assintomáticas, o que dificulta o diagnóstico e a percepção dos pacientes a respeito do seu quadro clínico. Em muitas situações os pacientes só têm a percepção de sintomas quando há um agravamento da doença (Brasil, 2013b).

Nesse contexto, a equipe de atenção básica, sobretudo os enfermeiros, tem indispensável papel na busca ativa dos pacientes para realização de consultas de rotina, bem como, na educação em saúde promovida a estes pacientes, tanto com o objetivo da promoção da saúde e prevenção da doença, quanto na educação e formulação do plano de cuidados a fim de prevenir agravamentos decorrentes dessa doença (Brasil, 2012b).

\section{Conclusão}

Pesquisas evidenciam que a disposição e a prática do perdão provocam transformações emocionais, físicas e fisiológicas. Em contrapartida, a ausência do perdão ocasiona estresse e sentimentos negativos, como raiva, rancor e mágoa. Tais sentimentos geram aumento da pressão arterial, frequência cardíaca e frequência respiratória. Diante disso, observa-se a necessidade do ato do perdão, especialmente em hipertensos, para a promoção do bem-estar físico, fisiológico e emocional.

Certamente, os objetivos deste estudo foram alcançados. Os resultados evidenciaram que os hipertensos se demostraram dispostos a perdoar. No presente estudo, houve uma prevalência de mulheres hipertensas, com idade superior a 60 anos e predomínio do estado civil casado. Além disso, o estudo mostrou que estado civil "casado" e "outros" interferem na disposição que o indivíduo tem de perdoar e que os hipertensos que residem com um a três indivíduos estão dispostos a perdoar mais. Apesar da idade não ter sido significativa estatisticamente, pode ser um dado que se relaciona com a ausência ou a disposição ao perdão, já que a maior parte da amostra possuía idade superior a 60 anos e indivíduos com idade avançada tende a perdoar mais, como ocorreu no presente estudo em que houve uma tendência ao perdão. Por fim, diante dos resultados obtidos, observa-se que os indivíduos hipertensos se demonstraram dispostos a perdoar.

Percebe-se, pelo estudo, que há relação entre fatores sociodemográficos, perdão e hipertensão arterial sistêmica. Ademais, verifica-se a necessidade do desenvolvimento de novas evidências científicas, a fim de melhorar a compressão dessas relações e sobretudo de aprimorar a assistência prestada aos hipertensos, observando-os nos diversos aspectos que interferem na manutenção da pressão arterial.

Por fim, espera- se que o estudo realizado sirva de encorajamento para o desenvolvimento de novas pesquisas acerca da temática abordada, visto que, o surgimento de novas evidências científicas promoverá melhor compreensão entre a 
disposição do perdão e hipertensão arterial sistêmica e em consequência disso, permitirá o aprimoramento da assistência prestada aos indivíduos hipertensos.

\section{Referências}

Alencar, T. F., \& Abreu, E. L. (2019, 29 de julho). O Perdão sob a Perspectiva do Ofensor: uma Revisão da Sistemática. Revista Psicologia: Ciência e Profissão, 39, 1-17. https://www.scielo.br/pdf/pcp/v39/1982-3703-pcp-39-e185662.pdf

Allemand, M. (2008, outubro). Age differences in forgivingness: The role of future time perspective. Journal of Research in Personality, 42 (5), 1137-1147. https://www.sciencedirect.com/science/article/abs/pii/S0092656608000287?via\%3Dihub

Andrade, T.F. (2014). Refinamento psicométrico da escala de atitudes para o perdão (efi) (Dissertação de mestrado). Universidade Federal da Paraíba, João Pessoa, PB.

Avezum, S. G. P. (2018). Avaliação da Disposição para o Perdão em Pacientes com Hipertensão Aguda do Miocárdio (Dissertação de Mestrado). Universidade Santo Amaro, São Paulo, SP.

Barbosa, E., Guimarães, J. I., \& Saraiva, R. Hipertensão Arterial Sistêmica e a Mulher. (2008, setembro). Revista da Sociedade de Cardiologia do Rio Grande do Sul, (15). http://sociedades.cardiol.br/sbc-rs/revista/2008/15/pdf/hipertensao_arterial.pdf

Barbosa, L. H. G. M. (2015). Explicando a disposição para perdoar: o papel dos valores humanos e das crenças no mundo justo/injusto (Dissertação de Mestrado). Universidade Federal da Paraíba, João Pessoa, PB.

Barroso, et al. (2021). Diretrizes Brasileiras de Hipertensão Arterial - 2020. Arquivos. Brasileiros de. Cardiologia, 116 (3), 516-658. https://abccardiol.org/wp-content/uploads/articles_xml/0066-782X-abc-116-03-0516/0066-782X-abc-116-03-0516.x44344.pdf

Basset, D. R. Jr., Fitzhugh, E. C., Crespo, C. J., King, G. A., \& McLaughlin, J. E. (2002, fevereiro). Physical activity and ethnic differences in hypertension prevalence in the United States. https://pubmed.ncbi.nlm.nih.gov/11817913/\#: :text=Background\%3A\%20In\%20the\%20United\%20States,hypertension\%20than\%20other\%20ethnic\%20grou ps.\&text=Results\%3A\%20Hypertension\%20prevalence\%20was\%20significantly,\%2C\%20CI\%200.59\%20to\%200.90)

Brasil. Ministério da Saúde. Secretaria de Educação Continuada, Alfabetização, Diversidade e Inclusão. (2012ª). Saúde Indígena: uma introdução ao tema. Série Vias dos Saberes. Brasília: Ministério da Saúde.

Brasil. Ministério da Saúde. Secretaria de Atenção à Saúde. Departamento de Atenção Básica. (2012b). Política Nacional de Atenção Básica. Brasília: Ministério da Saúde.

Brasil. Ministério da Saúde. Conselho Nacional de Saúde. (2013a). Resolução $n^{\circ}$ 466, de 12 de dezembro de 2012. Brasília: Ministério da Saúde.

Brasil. Ministério da Saúde. Secretaria de Atenção à Saúde. Departamento de Atenção Básica. (2013b). Estratégias para o cuidado da pessoa com doença crônica: hipertensão arterial sistêmica. Brasília: Ministério da Saúde.

Brasil. Ministério da Saúde. Conselho Nacional de Saúde. (2016). Resolução $n^{o} 510$ de 7 de abril de 2016. Brasília: Ministério da Saúde.

Brasil. Ministério da Saúde. Secretaria de Vigilância em Saúde (2019). Vigitel Brasil 2018: Vigilância de Fatores de Risco e Proteção para Doenças Crônicas por Inquérito Telefônico. Brasília: Ministério da Saúde.

Bueno, R. K., Souza, S. A., Monteiro, M. A. (2013, janeiro). Processo de diferenciação dos casais de suas famílias de origem. Psico, 44 (1), 16-25. https://revistaseletronicas.pucrs.br/ojs/index.php/revistapsico/article/view/9420/8843

Cesarino, C. B, et al. (2008, julho). Prevalência e Fatores Sociodemográficos em Hipertensos de São José do Rio Preto - SP. Arquivos Brasileiros de Cardiologia, $91 \quad$ (1). https://www.scielo.br/scielo.php?script=sci_arttext\&pid=S0066782X2008001300005\#: :text=A\%20preval\%C3\%AAncia\%20desses\%20fatores\%20sociodemogr\%C3\%A1ficos,o\%20risco\%20de\%20complica\%C3\%A7\%C $3 \% \mathrm{~B} 5$ es\%20cardiovasculares

DeShea, L. A. (2003). Scenario-based scale of willingness to forgive. Individual Differences Research, 1 (3), 201-217. https://www.researchgate.net/profile/Lise-Deshea/publication/232477938_A_Scenario-

Based_Scale_of_Willingness_to_Forgive/links/5693e09308ae820ff0729002/A-Scenario-Based-Scale-of-Willingness-to-Forgive.pdf

Ferreira, P. A. A., Bodevan, E. C., \& Oliveira, L. C. (2019). Características Sociodemográficas Associadas à Prevalência de hipertensão Arterial Sistêmica. Revista da Universidade Vale do Rio Verde, 17 (1), 1. http://periodicos.unincor.br/index.php/revistaunincor/article/view/5003

Fonsêca, P. N., et al. Perdão Conjugal: Uma Explicação a partir dos Valores Humanos. (2017, dezembro). Trends in Psychology, 25 (4). https://www.scielo.br/pdf/tpsy/v25n4/2358-1883-tpsy-25-04-1913.pdf

Ghaemmaghami, P., Allemand, M., \& Martin, M. (2011, 8 de setembro). Forgiveness in Younger, Middle-Aged and Older Adults: Age and Gender Matters. Journal of Adult Development, v.18, n.4, p. 192-203, 2011. https://link.springer.com/article/10.1007/s10804-011-9127-X

Gois, C. F. L., et al. (2016, 27 de junho). Perfil Sociodemográfico e Clínico de Hipertensos Atendidos por Equipe de Saúde da Família. REME- Rev Min Enferm, 20. http://www.reme.org.br/artigo/detalhes/1095

Gouveia, V.V., et. al. (2009). Disposição para perdoar, desejabilidade social e religião: um estudo correlacional. Revista Bioética, 17 (2), 297-308. https://revistabioetica.cfm.org.br/index.php/revista_bioetica/article/view/168 
Gouveia, V. V., et. al. (2015, junho). Escala de Disposição para Perdoar: estrutura, consistência interna e invariância fatorial. Estudos de psicologia, 32 (2). http://www.scielo.br/scielo.php?pid=S0103-166X2015000200151\&script=sci_abstract\&tlng=pt

Hajjar, I., Kotchen, J. M., \& Kotchen, T. A. (2006). Hypertension: trends in prevalence, incidence, and control. Annu Rev Public Health, 27, 465-490. https://pubmed.ncbi.nlm.nih.gov/16533126/\#: :text=Incidence\%20rates\%20of\%20hypertension\%20range,controlled\%20in\%20the\%20United \%20States

Köche, J. C. (2011). Fundamentos de Metodologia Científica: Teoria da ciência e iniciação à pesquisa. Petrópolis, Brasil: Editora Vozes.

Liu, H., et al. (2017, outubro). Association between duration of oral contraceptive use and risk of hypertension: A meta-analysis. J Clin Hypertens, 19, 10321041. https://pubmed.ncbi.nlm.nih.gov/28612347/

Lucena, et al. (2021, 12 de novembro). Desempenho dos Serviços da Atenção Primária à Saúde: Satisfação das Pessoas com Hipertensão. Revista Ciência, Cuidado e Saúde, v. 20. https://periodicos.uem.br/ojs/index.php/CiencCuidSaude/article/view/53086. Acesso em: 12 mar. 2021.

Mccullough, M.E., Bellah, C. G., Kilpatrick, S. D., \& Johnson, J. L. (2001, 1 de maio). Vengefulness: Relationships with Forgiveness, Rumination, WellBeing, and the Big Five. Personality and Social Psychology Bulletin, 27 (5), 601-610. https://journals.sagepub.com/doi/abs/10.1177/0146167201275008

Mccullough, M.E., \& Witvliet, C.V. (2001, 1 de janeiro). The Handbook of Positive Psychology. The Psychology of Forgiveness, 446-458. https://www.researchgate.net/publication/264443222_The_psychology_of_forgiveness

Mendonça, E.T., et al. (2015, dezembro). Perfil Sociodemográfico, Clínico e Cardiovascular Adicional de Indivíduos Hipertensos. Revista de enfermagem UFPE online, 9 (12), 1182-1189. https://periodicos.ufpe.br/revistas/revistaenfermagem/article/view/10823

Ong, K.L. Cheung, B. M. Y., Man, Y. B., Lau, C. P., \& Lam, K. S, L. (2007, janeiro). Prevalence, awareness, treatment, and control of hypertension among United States adults 1999- 2004. Journal of the American Heart Association, 49 (1), 69-75. https://pubmed.ncbi.nlm.nih.gov/17159087/

Organização Pan-Americana de Saúde. Organização Mundial da Saúde. (2016). Dia Mundial da Hipertensão 2016. Brasília: OPAS/OMS.

Organização Pan-Americana de Saúde. Organização Mundial da Saúde. (2020). Histórico da pandemia de COVID-19. Brasília: OPAS/OMS.

Pereira, V.N.A. (2013). Religiosidade em Indivíduos Hipertensos de uma Unidade do Programa Saúde da Família de Pedras de Fogo - PB (Dissertação de Mestrado). Universidade Federal da Paraíba, João Pessoa, PB.

Pinho, V. D., \& Falcone, E. M. O. (2015, agosto). Intervenciones para la Promoción del Perdón y la Inserción de la Empatía: Revisión de la Literatura. Revista Argentina de Clínica Psicológica, 24 (2), 111-120. https://www.redalyc.org/pdf/2819/281946783003.pdf

Pinho, V. D., \& Falcone, E. M. O. (2018, janeiro). Estudo qualitativo sobre fatores facilitadores e dificultadores do perdão interpessoal. Psicologia Social, 18, (1), 189-208. http://pepsic.bvsalud.org/pdf/epp/v18n1/v18n1a11.pdf

Rye, M.S., et. al. (2001). Evaluation of the Psychometric Properties of Two Forgiveness Scales. Current Psychology: Developmental, Learning, Personality, Social Spring, 20 (3), 260-277. https://greatergood.berkeley.edu/images/uploads/Rye-Forgiveness_Scale_Psychometric_Comparison.pdf

Salmazi, C. (2015). Cura pelo Perdão. Recuperado de http://www.ippb.org.br/index.php?option=com_content\&view=article\&id=3705\&catid= 80:mythos\&Itemid $=110$

Santana, R. G. (2011). Estudo das Relações entre a Atitude de Perdoar Ofensas Interpessoais e os Esquemas Iniciais Desadaptativos (Dissertação de Mestrado). Universidade Federal de Uberlândia, Uberlândia, MG.

Santana, R.G., \& Lopez, R. F. F. (2012). Aspectos Conceituais do Perdão no Campo da Psicologia. Psicologia: Ciência e Profissão, 32 (3), 618-631. https://www.scielo.br/scielo.php?pid=S1414-98932012000300008\&script=sci_abstract\&tlng=pt

Silva, C. F. S., et al. (2016). Espiritualidade e religiosidade em pacientes com hipertensão arterial sistêmica. Rev. bioét., 24 (2). https://www.scielo.br/pdf/bioet/v24n2/1983-8034-bioet-24-2-0332.pdf

Silva, G. C. (2020). Pesquisa de opinião sobre o perdão: qual a sua relevância e como os indivíduos o percebem (Trabalho de Conclusão de Curso), Universidade Federal De Uberlândia, Uberlândia, MG.

Sociedade Brasileira de Cardiologia. Sociedade Brasileira de Hipertensão. Sociedade Brasileira de Nefrologia. (2010). VI Diretrizes Brasileiras de Hipertensão. Rio de Janeiro: Sociedade Brasileira de Cardiologia. Sociedade Brasileira de Hipertensão. Sociedade Brasileira de Nefrologia.

Malachias, M. V. B., et al. (2016, setembro). $\quad 7^{\text {a }}$ Diretriz Brasileira de Hipertensão Arterial. Arq Bras Cardiol, 107 (3), 1-103. http://publicacoes.cardiol.br/2014/diretrizes/2016/05_HIPERTENSAO_ARTERIAL.pdf

Steiner, M., Allemand, \& M., Mccullough, M.E. (2011, 5 de dezembro). Do agreeableness and neuroticism explain age differences in the tendency to forgive others?. Personality and Social Psychology Bulletin, 38 (4), 441-453. https://journals.sagepub.com/doi/10.1177/0146167211427923

Toledo, N. N., et. al. (2020). Fatores de risco cardiovascular: diferenças entre grupos étnicos. Revista Brasileira de Enfermagem, 73 (4). https://www.scielo.br/pdf/reben/v73n4/pt_0034-7167-reben-73-04-e20180918.pdf

Tsang, J.Á., Mccullough, M.E., \& Hoyt, W.T. (2005). Psychometric and rationalization accounts of the religion-forgiveness discrepancy. Journal of Social Issues, 61 (4), 785-805. http://www.baylorisr.org/wp-content/uploads/tsang_psychometric.pdf

Worthington, E.L., \& Scherer, M. (2004, janeiro). Forgiveness is an Emotion-Focused Coping Strategy That Can Reduce Health Risks and Promote Health Resilience: Theory, Review, and Hypotheses. Psychology and Health, 19 (3), 385-405. https://www.researchgate.net/publication/247500255 Forgiveness is an emotionfocused_coping_strategy_that_can_reduce_health_risks_and_promote_health_resilience_Theory_review_and_hypotheses 
Research, Society and Development, v. 10, n. 10, e585101019174, 2021

(CC BY 4.0) | ISSN 2525-3409 | DOI: http://dx.doi.org/10.33448/rsd-v10i10.19174

Worthington, E. L., et al. (2015, janeiro). Forgiveness-Reconciliation and CommunicationConflict-Resolution Interventions Versus Retested Controls in Early Married Couples. J Couns Psychol, 62 (1), 14-27. https://pubmed.ncbi.nlm.nih.gov/25264599/

Zaitune, M. P. A., Barros, M. B. A., César, C. L. G., Carandina, L., \& Goldbaum, M. Hipertensão arterial em idosos: prevalência, fatores associados e práticas de controle no Município de Campinas. Cad. Saúde Pública, 22 (2), 285-294. https://www.scielo.br/pdf/csp/v22n2/06.pdf 